\title{
Oral Administration of Chitosan Attenuates Bleomycin-induced Pulmonary Fibrosis in Rats
}

\author{
YOU-SEOK KIM ${ }^{1,2}$, QIANG LI ${ }^{1}$, HWA-YOUNG YOUN ${ }^{1}$ and DAE YOUNG KIM ${ }^{3}$ \\ ${ }^{1}$ Department of Veterinary Internal Medicine, College of Veterinary Medicine, \\ Seoul National University, Seoul, Republic of Korea, \\ ${ }^{2}$ KPC Corporation, Oporo, Gwangju, Republic of Korea; \\ ${ }^{3}$ Department of Life Science, College of Bio-nano Technology, Gachon University, Seongnam, Republic of Korea
}

\begin{abstract}
Background/Aim: Idiopathic pulmonary fibrosis $(P F)$ is a fatal disorder of unknown aetiology with limited treatment options. Chitosan has antibacterial, antifungal, antioxidant, antitumour, and anti-inflammatory effects. This study aimed to investigate the effects of chitosan administration on bleomycin (BLM)-induced PF in rats. Materials and Methods: A PF rat model was established by endotracheal instillation of $5 \mathrm{mg} / \mathrm{kg} B L M$; then, chitosan was administered in drinking water for 3 weeks. Histology, cell counts, and cytokine responses in the bronchoalveolar lavage fluid (BALF) and weight measurements (body and lung) were analyzed to assess its therapeutic effects. Results: Chitosan administration tended to reduce transforming growth factor (TGF)- $\beta 1$ and interferon (IFN)- $\gamma$ levels in $B A L F$, and histopathological examination confirmed that chitosan attenuated the degree of inflammation and fibrosis in the lung. Conclusion: This study revealed that oral chitosan exhibits potential antifibrotic effects, as measured by decreased proinflammatory cytokine levels and histological evaluation, in a BLM-induced PF rat model.
\end{abstract}

Pulmonary fibrosis $(\mathrm{PF})$ is a chronic, progressive lung disease characterised by the excessive proliferation of fibroblasts and deposition of collagen (fibrosis) in the pulmonary interstitium $(1,2)$. Despite extensive investigations, the cause and pathogenesis of PF remain unclear (3). Currently available therapies for PF include corticosteroids, immunosuppressants,

This article is freely accessible online.

Correspondence to: Dae Young Kim, Department of Life Science, College of Bio-nano Technology, Gachon University, 1342 Seongnamdaero, Sujeong-gu, Seongnam-si, Gyeonggi-do 13120, Republic of Korea. Tel: +82 317504761, e-mail: davekim@gachon.ac.kr

Key Words: Bleomycin, chitosan, oral administration, pulmonary fibrosis. immunomodulators, antioxidants, antileukotrienes, antifibrotics, oxygen therapy, and lung transplantation (4). However, these therapies are unsatisfactory and incapable of reversing lung damage $(2,4)$. Therefore, it is crucial to find effective and nontoxic therapeutic strategies for PF.

Chitosan, both of natural and synthetic origin, is partially $\mathrm{N}$-deacetylated chitin. Chitin is a linear homopolymer of 1,4linked N-acetyl-D-glucosamine, which is obtained from chitinrich crab shell (5). Chitosan exhibits beneficial effects because of its biological properties, which include antitumour, antiangiogenetic, antibacterial, anti-inflammatory, antioxidant, and antidiabetic properties $(4,6)$. Chitosan also has antifibrotic effects, such as reduction of fibroblast infiltration and fibrosis, as well as degradation of collagen (5, 7-9). Moreover, chitosan aerosol inhalation has shown antifibrotic effects in a PF-rat model (4). In this experiment, we used oral administration of chitosan (rather than aerosol inhalation) for convenience, in order to examine its antifibrotic effects in rats with bleomycin (BLM)-induced PF.

\section{Materials and Methods}

Animals and experimental design. This study was approved by the Institutional Animal Care and Use Committee of KPC Co., in accordance with the Animal Protection Act (P170050). Pathogen-free male Sprague-Dawley rats were obtained from the Orient Bio Co. (Seoul, Republic of Korea). The rats were housed in a controlled environment with a 12-h light/dark cycle and standard food and water ad libitum. They were divided into 4 groups as follows: a sham group $(\mathrm{G} 1 ; \mathrm{n}=6)$ with intratracheal (IT) administration of saline at day 0 only, along with $2 \%$ vehicle solution without chitosan (instead of drinking water); negative control group (G2; $n=6)$ with intratracheal administration of BLM at day 0 only, along with $2 \%$ vehicle solution without chitosan (instead of drinking water); positive control group ( $\mathrm{G} 3 ; \mathrm{n}=6)$ with intratracheal administration of BLM at day 0 only, along with prednisolone (PDS, $5 \mathrm{mg} / \mathrm{kg}$ ) by gavage daily; and test group $(\mathrm{G} 4, \mathrm{n}=6)$ with intratracheal administration of BLM at day 0 only, along with $2 \%$ chitosan solution (instead of drinking water). All test and vehicle solutions were administered beginning on day 0 and ending on day 20 . 
Preparation of chitosan. High-molecular-weight chitosan (approximately $100 \mathrm{kDa}$ ) with a degree of deacetylation (approximately 92\%) was purchased from YB Bio (Yeongdeok-gun, Republic of Korea) and converted to medium-molecular-weight chitosan using a previously reported method (10). The prepared chitosan solution was dissolved in drinking water at a concentration of $2 \%(\mathrm{w} / \mathrm{v})$ to prepare the test agent, which was then administered orally to the test group.

Induction of lung fibrosis. After 1-week acclimatization, the rats were anesthetised with isoflurane (Isotroy, Troikaa Pharmaceuticals, Ahmedabad, India) inhalation and then administered $5 \mathrm{mg} / \mathrm{kg}$ of BLM (Bleomycin, Dong-A ST, Seoul, Republic of Korea) once at a dose of $1 \mathrm{ml} / \mathrm{kg}$ by intratracheal instillation. The rats in the sham group were treated with saline once via the same administration route described above. PDS was administered once daily for 3 weeks, starting at day 0 after the BLM administration; chitosan and vehicle were administered continuously in drinking water, beginning on day 0 and ending on day 20 . At the end of this period, the rats were sacrificed, and autopsies were performed.

Body weight and lung weight. Body weights were measured every 7 days during the experiment period. Prior to sacrifice, all rats were fasted for approximately $16 \mathrm{~h}$, with water intake permitted. At the time of autopsy, body weights were measured. The lungs were removed during autopsy and the absolute lung weights were measured; relative lung weights were then calculated as the ratio of lung weight to body weight.

Bronchoalveolar lavage (BAL). BAL fluid (BALF) was collected by intratracheal instillation of $5 \mathrm{ml}$ of sterile saline into the lungs. Whole BALF was then centrifuged at $1500 \mathrm{rpm}$ for $5 \mathrm{~min}$. After centrifugation, the residual suspension was analysed to determine the total cell, neutrophil, macrophage, and lymphocyte counts using a haematology analyser (Advia, Siemens, Munich, Germany). The supernatant was analysed for multiple pro-inflammatory cytokines, namely tumour necrosis factor (TNF)- $\alpha$, transforming growth factor (TGF)- $\beta 1$, interferon (IFN)- $\gamma$, interleukin (IL)-4, and IL-6 using ELISA kits (Elabscience, Houston, TX, USA), in accordance with the manufacturer's instructions.

Lung tissue histopathology. After BALF collection, lung tissue specimens were fixed in $10 \%$ neutral buffered formalin, then trimmed, dehydrated, and embedded in paraffin, in accordance with standard procedures for histological examination (11). The prepared paraffinembedded blocks were sectioned into $4-\mu \mathrm{m}$ thick slides and stained with haematoxylin and eosin (H\&E) using Masson's trichrome to assess the severity of alveolitis and PF. For this assessment, a semiquantitative grading system was used based on the following established scale (12): Grade 0, no alveolitis/fibrosis was observed; grade 1 (mild), focal lesions occupying $<25 \%$ or $<20 \%$ (for alveolitis and fibrosis, respectively) of the lung were detected in the alveolar septum; grade 2 (moderate), widespread alveolitis or fibrosis involving $2550 \%$ or $2050 \%$, respectively, of the lung was observed; and grade 3 (severe), a diffused alveolitis or fibrosis spanning $>50 \%$ of the lung was observed, with occasional consolidation of air spaces and patches of haemorrhagic areas within the interstitium.

Statistical analysis. Data were analysed using SPSS (version 20, IBM SPSS Statistics, Chicago, IL, USA). A one-way analysis of variance was used to determine the differences among groups, followed by a post hoc test (LSD or Dunnett T3) to determine which groups were significantly different from G2.

\section{Results}

Body weight and lung tissue weight. During the experimental period, no significant changes in body weight were observed in any of the groups when compared to G2. However, G4 showed less prominent propensity for weight loss than G2 and G3 during the total experiment period when compared to G1 (Figure 1). After BLM-induced PF, the absolute and relative lung weights in $\mathrm{G} 1$ were significantly lower than those in $\mathrm{G} 2(p<0.01, p<0.05)$ (Figure $2 \mathrm{~A}$ and $\mathrm{B})$.

Cell counts in BALF. Analysis of cells in the BALF revealed a significantly higher neutrophil count in G3 than in G2 $(p<0.05)$ (Table I).

Concentrations of cytokines in the BALF. When levels of TNF- $\alpha$, TGF- $\beta 1$, IFN- $\gamma$, IL- $1 \beta$, IL-4, and IL-6 were measured in BALF supernatants, there were no significant differences observed among the groups. However, the levels of cytokines in the BALF were lower in G4 than in G2 (Table II).

Histological evaluation of lung tissues. When the inflammatory status was assessed in lung tissues stained with H\&E, the inflammation scores were significantly lower in G1 and G4 $[0.33 \pm 0.21(p<0.01)$ and $1.50 \pm 0.34(p<0.05)$, respectively] than in $\mathrm{G} 2(2.50 \pm 0.22)$. Comparison of fibrosis in lung tissues stained with Masson trichrome also revealed significantly less lung fibrosis in G1 and G4 [fibrosis scores of $0.17 \pm 0.17$ $(p<0.01)$ and $1.33 \pm 0.33(p<0.05)$, respectively] than in $\mathrm{G} 2$

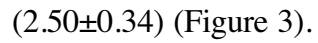

\section{Discussion}

The present study was conducted to examine the antifibrotic effects of orally administered chitosan in a rat model of BLM-induced PF. It has been reported that BLM administered intratracheally to induce PF can reduce body weight while increasing lung weight, although these changes can be improved with suitable medication (13). Lung weight increases in proportion to the dose of intratracheally administered BLM, and the increased lung weight is associated with histopathological fibrosis scores (14).

The present study demonstrated that there was a tendency for body weight loss during the first week after BLM administration in the negative control group, whereas the chitosan group showed reduced propensity for weight loss during the same period. Significantly increased lung weight was also found in the negative control group, relative to that in the sham group. However, the chitosan group showed a 
A

$\begin{array}{ll}\text { IT saline + Vehicle solution }(n=6) & \rightarrow \text { G1 (IT Saline+Vehicle) } \\ \text { IT BLM + Vehicle solution }(n=6) & \rightarrow \text { G2 (IT BLM+Vehicle) } \\ \text { IT BLM + PDS }(n=6) & \rightarrow \text { G3 (IT BLM+PDS) } \\ \text { IT BLM + Chitosan }(n=6) & \text { G4(IT BLM+Chitosan) }\end{array}$

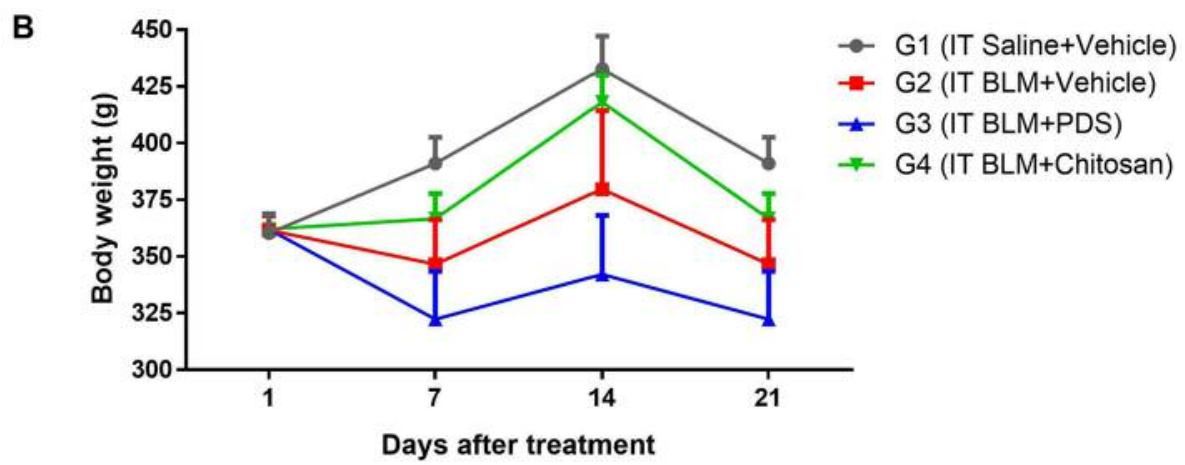

Figure 1. Experimental groups (A). Body weights were measured for each group during the 21-day experimental period (B). There was no significant difference in body weight among the groups. Data are expressed as mean \pm SE. IT, Intratracheal; BLM, bleomycin; PDS, prednisolone; SE, standard error.

Table I. Total numbers of cells, neutrophils, lymphocytes, and macrophages in the bronchoalveolar lavage fluid (BALF). The neutrophil count was significantly lower in the prednisolone (PDS)-treated group (G3) than in the negative control group (G2).

\begin{tabular}{|c|c|c|c|c|c|}
\hline \multirow[t]{2}{*}{ Group } & \multirow[t]{2}{*}{ Result } & \multicolumn{4}{|c|}{ Cell count in BALF } \\
\hline & & $\begin{array}{c}\text { Total } \\
\left(\times 10^{3} \text { cell } / \mu \mathrm{l}\right)\end{array}$ & $\begin{array}{l}\text { Neutrophils } \\
\left(\times 10^{3} \text { cell } / \mu \mathrm{l}\right)\end{array}$ & $\begin{array}{l}\text { Lymphocytes } \\
\left(\times 10^{3} \text { cell } / \mu \mathrm{l}\right)\end{array}$ & $\begin{array}{l}\text { Macrophages } \\
\left(\times 10^{3} \text { cell } / \mu \mathrm{l}\right)\end{array}$ \\
\hline \multirow[t]{3}{*}{ G1 (IT Saline + Vehicle) } & Mean & 121.7 & 33.6 & 79.1 & 2.5 \\
\hline & SE & 28.9 & 6.6 & 21.3 & 0.8 \\
\hline & $\mathrm{N}$ & 6 & 6 & 6 & \\
\hline \multirow[t]{3}{*}{ G2 (IT BLM + Vehicle) } & Mean & 346.7 & 36.5 & 298.0 & 4.0 \\
\hline & SE & 192.9 & 10.8 & 179.8 & 1.9 \\
\hline & $\mathrm{N}$ & 6 & 6 & 6 & \\
\hline \multirow[t]{3}{*}{ G3 (IT BLM + PDS) } & Mean & 1065.0 & $116.3^{*}$ & 900.0 & 12.5 \\
\hline & SE & 502.0 & 18.9 & 478.7 & 4.8 \\
\hline & $\mathrm{N}$ & 6 & 6 & 6 & \\
\hline \multirow[t]{3}{*}{ G4 (IT BLM + Test article) } & Mean & 295.0 & 58.9 & 212.7 & 3.9 \\
\hline & SE & 25.3 & 4.9 & 24.8 & 0.8 \\
\hline & $\mathrm{N}$ & 6 & 6 & 6 & \\
\hline
\end{tabular}

BLM, Bleomycin; SE, standard error. * $p<0.05$ compared to the G2 group.

tendency for a reduction in lung weight. Thus, chitosan tended to show protective potential against the increase in lung weight.

While fibrosis progression is characterised by extracellular matrix (ECM) accumulation and fibroblast proliferation, chitosan is known to inhibit the latter with potent effects on collagen synthesis and ECM production (4, 15). TGF- $\beta$ reportedly plays a crucial role in idiopathic PF and animal models of lung fibrosis (16-18). The present study showed a tendency for higher levels of TGF- $\beta 1$ in the BALF in the negative control group, relative to those in the sham group. 
A

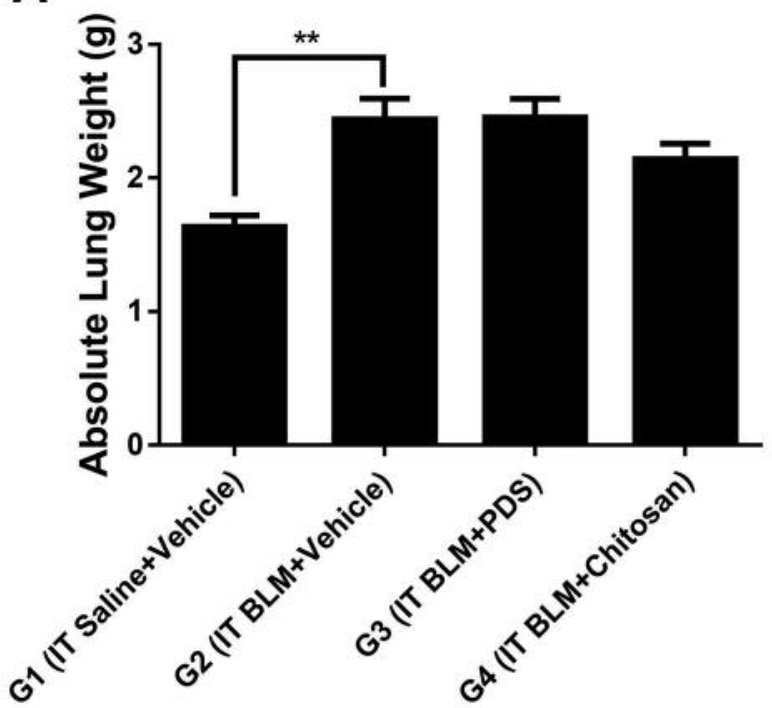

B

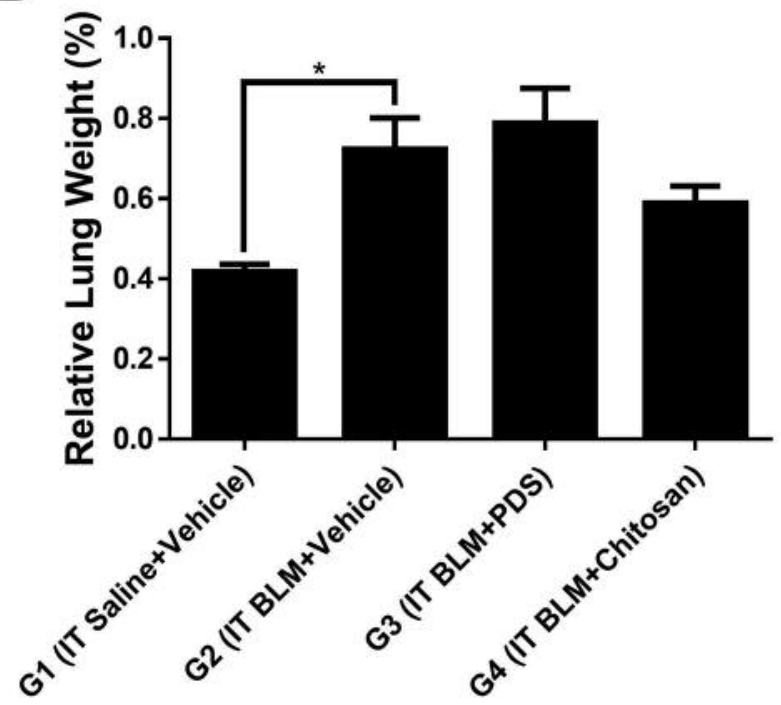

Figure 2. Absolute $(A)$ and relative $(B)$ weights of the lungs measured during autopsy. The lung weight was significantly lower in the sham group (G1) than in the negative control group $(G 2) .{ }^{*} p<0.05,{ }^{*} p<0.01$. Data are expressed as mean $\pm S E$. IT, Intratracheal; BLM, bleomycin; PDS, prednisolone; SE, standard error.

Table II. Cytokine concentrations in the bronchoalveolar lavage fluid. No group showed a significant difference compared with the negative control group (G2).

\begin{tabular}{|c|c|c|c|c|c|c|}
\hline \multirow[t]{2}{*}{ Group } & \multirow[t]{2}{*}{ Results } & \multicolumn{4}{|c|}{ Cytokine level } & \multirow[b]{2}{*}{ IL-6 (pg/ml } \\
\hline & & TNF- $\alpha(\mathrm{pg} / \mathrm{ml})$ & TGF- $\beta 1$ (ng/ml) & $\mathrm{IFN}-\gamma(\mathrm{pg} / \mathrm{ml})$ & IL-4 (pg/ml) & \\
\hline \multirow[t]{3}{*}{ G1 (IT Saline + Vehicle) } & Mean & 54.51 & 8.05 & 0.39 & 6.83 & 18.99 \\
\hline & SE & 25.40 & 2.42 & 0.39 & 5.60 & 12.20 \\
\hline & $\mathrm{N}$ & 6 & 6 & 6 & 6 & 6 \\
\hline \multirow[t]{3}{*}{ G2 (IT BLM + Vehicle) } & Mean & 47.05 & 24.35 & 8.45 & 6.51 & 44.20 \\
\hline & SE & 20.13 & 8.75 & 5.62 & 2.93 & 21.83 \\
\hline & $\mathrm{N}$ & 6 & 6 & 6 & 6 & 6 \\
\hline \multirow[t]{3}{*}{ G3 (IT BLM + PDS) } & Mean & 38.14 & 11.73 & 3.08 & 1.55 & 23.24 \\
\hline & SE & 15.60 & 2.17 & 2.00 & 0.99 & 9.58 \\
\hline & $\mathrm{N}$ & 6 & 6 & 6 & 6 & 6 \\
\hline \multirow{3}{*}{ G4 (IT BLM + Test article) } & Mean & 2.93 & 8.84 & 0.99 & 2.04 & 6.50 \\
\hline & SE & 2.93 & 1.72 & 0.99 & 1.29 & 6.50 \\
\hline & $\mathrm{N}$ & 6 & 6 & 6 & 6 & 6 \\
\hline
\end{tabular}

BLM, Bleomycin; IFN, interferon; IL, interleukin; TGF, transforming growth factor; TNF, tumour necrosis factor; PDS, prednisolone; SE, standard error.

Furthermore, the chitosan group showed a tendency for reduction in TGF- $\beta 1$ expression, thus approaching a level similar to that in the sham group. Importantly, TNF- $\alpha$ is involved in proinflammatory and profibrotic activity, as well as ECM remodelling, in idiopathic PF (19). IL-4 is also known to stimulate fibroblast chemotaxis, proliferation, collagen synthesis, and myofibroblast differentiation in vitro; however, its in vivo role has not been clearly established (20,
21). IFN- $\gamma$ can alleviate the degree of PF by antagonizing the profibrotic action of TGF- $\beta 1(21,22)$. However, these positive effects of IFN- $\gamma$ have not been induced in clinical settings, according to previous studies (21). Furthermore, IL6 has been found to act as both a proinflammatory and profibrotic factor and a protective factor in relation to PF (23). During fibrosis progression, the functions of different cytokines vary on the basis of illness stage (acute vs. 
A
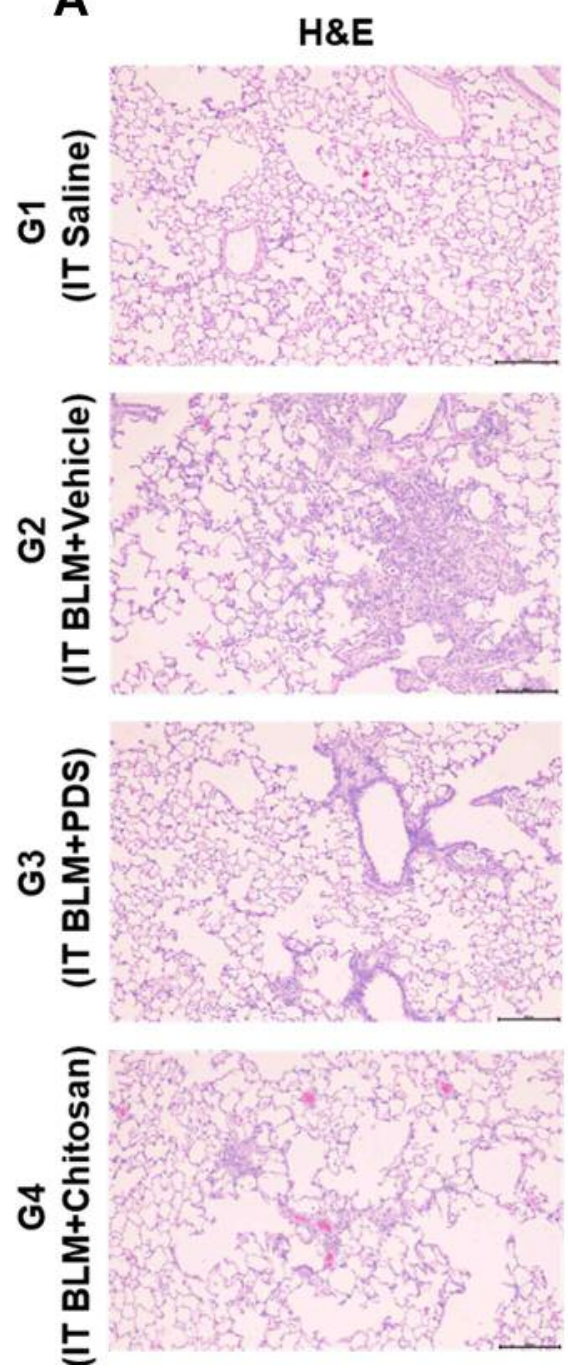

Masson's trichrome
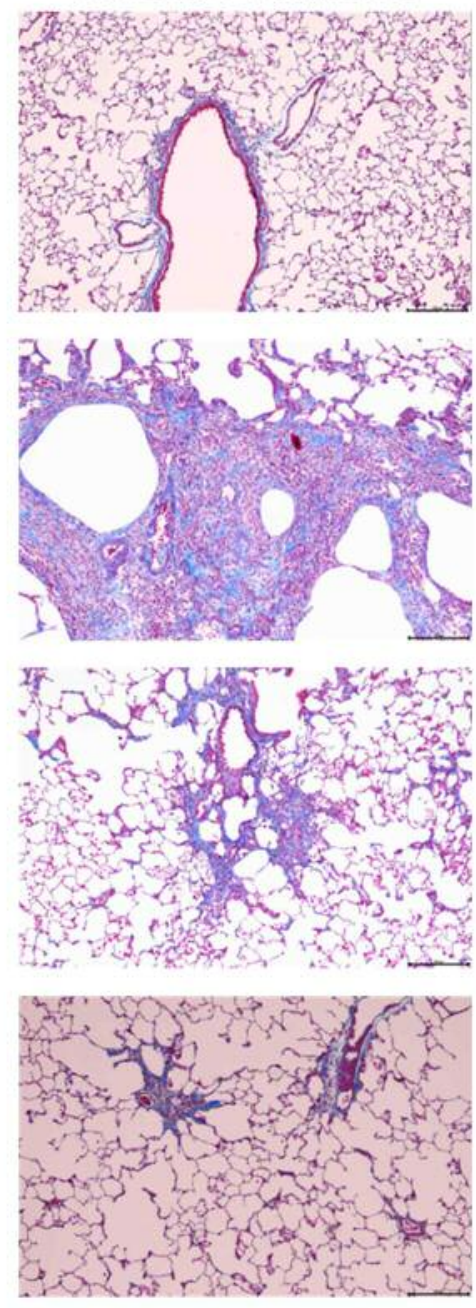

B

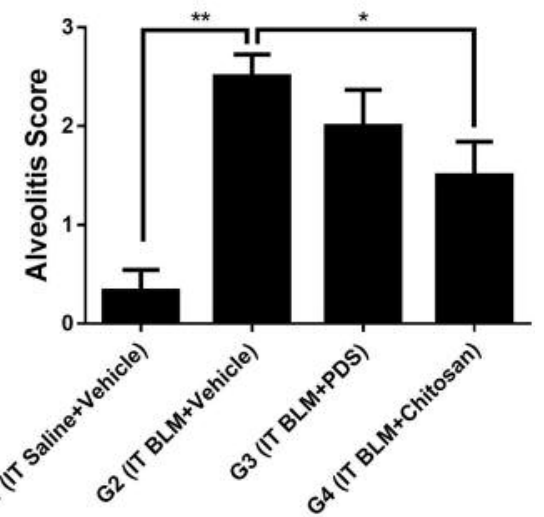

C

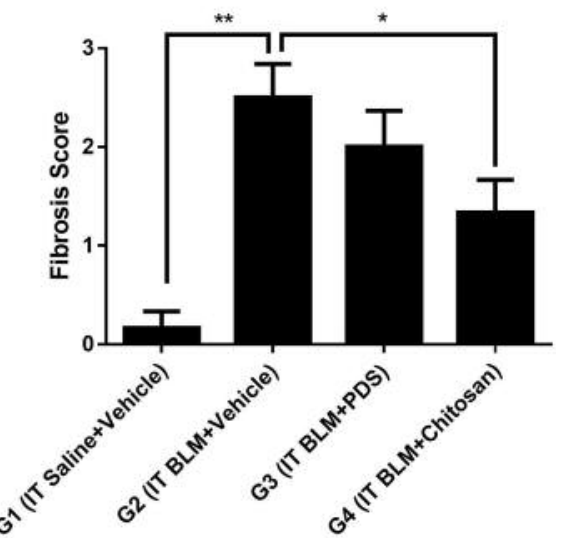

Figure 3. At 21 days post treatment, the lung tissues of the rats in each group were stained with haematoxylin and eosin (H\&E) and Masson's trichrome. (A) The sham group (G1) showed normal histological structures with both stains. However, the negative control group (G2) showed severe alveolitis and fibrosis (A). Magnification $\times 100$; scale bar $200 \mu \mathrm{m}$. Inflammation score (B) and fibrosis score (C) were less in the sham (G1) and chitosan (G4) groups than in the negative control group $(G 2) .{ }^{*} p<0.05,{ }^{*} p<0.01$. Data are expressed as mean $\pm S E$. IT, Intratreacheal; BLM, bleomycin; PDS, prednisolone; SE, standard error.

chronic), location of intrapulmonary lesion (bronchial vs. alveolar), cellular level (epithelial vs. fibroblast), and immune status (inflammatory $v s$. immunosuppressive) (24).

In the present study, analysis of TGF- $\beta 1$, TNF- $\alpha$, IFN- $\gamma$, IL-4, and IL-6 levels in the BALF revealed lower concentrations of these cytokines in the chitosan group than in the negative control group. Moreover, the concentrations of TGF- $\beta 1$, IFN- $\gamma$, and IL- 6 were lower in the sham than in the negative control group. However, the concentrations of TNF- $\alpha$ and IL-4 were higher in sham group than in negative control group. These results are supported by earlier findings that the immune response in the development of fibrosis is affected by compound factors, rather than a single factor, and that the contribution of cytokines varies in lung fibrosis. It is possible that the results of this study could have been affected by the timing of sampling conducted according to research design and type of samples (e.g., serum, BALF, and tissues). Therefore, future studies can provide further assessment on the effects of chitosan administration on a PF model by addressing the many variables, such as samples and illness period. Furthermore, the histopathology changes of lung tissue are the most intuitive and gold standard for the 
evaluation of BLM-induced model $(25,26)$. The chitosan treated group (G4) showed a marked decrease in the degree of alveolitis scores and fibrosis score, compared to the negative control group (G2).

\section{Conclusion}

The present study demonstrated potential antifibrotic effects of orally administered chitosan, quantified by the levels of proinflammatory cytokines and histological evaluation, in an experimental model of BLM-induced PF.

\section{Conflicts of Interest}

There are no conflicts of interest of declare regarding this study.

\section{Authors' Contributions}

QL, HYY: Conception and design, collection and/or assembly of data, data analysis and interpretation; YSK, DYK: collection and/or assembly of data conception and design, data analysis and interpretation, manuscript writing, final approval of manuscript

\section{Acknowledgments}

The Authors would like to express their sincere gratitude to Prof. Park Jae-kweon, Department of Life Science, Gachon University, for providing them with chitosan.

\section{References}

1 Zhou XM, Cao ZD, Xiao N, Shen Q and Li JX: Inhibitory effects of amines from Citrus reticulata on bleomycin-induced pulmonary fibrosis in rats. Int J Mol Med 37(2): 339-346, 2016. PMID: 26675886. DOI: 10.3892/ijmm.2015.2435

2 Liu W, Wan J, Han JZ, Li C, Feng DD, Yue SJ, Huang YH, Chen Y, Cheng QM, Li Y and Luo ZQ: Antiflammin-1 attenuates bleomycin-induced pulmonary fibrosis in mice. Respir Res 14: 101, 2013. PMID: 24098933. DOI: 10.1186/1465-9921-14-101

3 Wuyts WA, Willems S, Vos R, Vanaudenaerde BM, De Vleeschauwer SI, Rinaldi M, Vanhooren HM, Geudens N, Verleden SE, Demedts MG, Thomeer M, Verbeken EK and Verleden GM: Azithromycin reduces pulmonary fibrosis in a bleomycin mouse model. Exp Lung Res 36(10): 602-614, 2010. PMID: 20874225. DOI: 10.3109/01902148.2010.492895

4 Zhou LL, He XY, Xu FY, Bu DX, Zou Z and Shi XY: Chitosan aerosol inhalation alleviates lipopolysaccharide induced pulmonary fibrosis in rats. Exp Lung Res 40(9): 467-473, 2014. PMID: 25322333. DOI: 10.3109/01902148.2014.948231

5 Ishihara M, Ono K, Sato M, Nakanishi K, Saito Y, Yura H, Matsui T, Hattori H, Fujita M, Kikuchi M and Kurita A: Acceleration of wound contraction and healing with a photocrosslinkable chitosan hydrogel. Wound Rep Regen 9(6): 513-521, 2001. PMID: 11896994.

6 Ngo DH, Vo TS, Ngo DN, Kang KH, Je JY, Pham HND, Byun HG and Kim SK: Biological effects of chitosan and its derivatives. Food Hydrocolloids 51: 200-216, 2015. DOI: 10.1016/j.foodhyd 2015.05 .023
7 Diegelmann RF, Dunn JD, Lindblad WJ and Cohen IK: Analysis of the effects of chitosan on inflammation, angiogenesis, fibroplasia, and collagen deposition in polyvinyl alcohol sponge implants in rat wounds. Wound Repair Regen 4(1): 48-52, 1996. PMID: 17129347. DOI: 10.1046/j.1524-475X.1996.40109.x

8 Hamano $\mathrm{T}$, Teramoto A, Iizuka $\mathrm{E}$ and Abe $\mathrm{K}$ : Effects of polyelectrolyte complex (PEC) on human periodontal ligament fibroblast (HPLF) function. II. Enhancement of HPLF differentiation and aggregation on PEC by L-ascorbic acid and dexamethasone. J Biomed Mater Res 41(2): 270-277, 1998. PMID: 9638532.

9 Chatelet C, Damour O and Domard A: Influence of the degree of acetylation on some biological properties of chitosan films. Biomaterials 22(3): 261-268, 2001. PMID: 11197501.

10 Lee CG and Park JK: Comparison of inhibitory activity of bioactive molecules on the dextransucrase from Streptococcus mutans. Appl Microbiol Biotechnol 99(18): 7495-7503, 2015. PMID: 26016785. DOI: 10.1007/s00253-015-6693-Z

11 Bancroft JD and Gamble M: Theory and practice of histological techniques. 6th edition. London, Churchill Livingstone, p150, 2008.

12 Zuo WL, Zhao JM, Huang JX, Zhou W, Lei ZH, Huang YM, Huang YF and Li HG: Effect of bosentan is correlated with MMP-9/TIMP-1 ratio in bleomycin-induced pulmonary fibrosis. Biomed Rep 6(2): 201-205, 2017. PMID: 28357073. DOI: 10.3892/br.2016.832

13 Abidi A, Aissani N, Sebai H, Serairi R, Kourda N and Ben Khamsa S: Protective effect of Pistacia lentiscus oil against bleomycin-induced lung fibrosis and oxidative stress in rat. Nutr Cancer 69(3): 490-497, 2017. PMID: 28287322. DOI: 10.1080/ 01635581.2017 .1283423

14 Gilhodes JC, Julé Y, Kreuz S, Stierstorfer B, Stiller D and Wollin L: Quantification of pulmonary fibrosis in a bleomycin mouse model using automated histological image analysis. PLoS One 12(1): e0170561, 2017. PMID: 28107543. DOI: 10.1371/ journal.pone. 0170561

15 de Jesús Valle MJ, Dinis-Oliveira RJ, Carvalho F, Bastos ML and Sánchez Navarro A: Toxicological evaluation of lactose and chitosan delivered by inhalation. J Biomater Sci Polym Ed 19(3): 387-397, 2008. PMID: 18325238. DOI: 10.1163/1568 56208783721038

16 Gross TJ and Hunninghake GW: Idiopathic pulmonary fibrosis. N Eng1 J Med 345(7): 517-525, 2001. PMID: 11519507. DOI: 10.1056/NEJMra003200

17 Li YJ, Azuma A, Usuki J, Abe S, Matsuda K, Sunazuka T, Shimizu T, Hirata Y, Inagaki H, Kawada T, Takahashi S, Kudoh S and Omura S: EM703 improves bleomycin-induced pulmonary fibrosis in mice by the inhibition of TGF-beta signaling in lung fibroblasts. Respir Res 7: 16, 2006. PMID: 16438734. DOI: 10.1186/1465-9921-7-16

18 Denton CP, Zheng B, Evans LA, Shi-wen X, Ong VH, Fisher I, Lazaridis K, Abraham DJ, Black CM and de Crombrugghe B: Fibroblast-specific expression of a kinase-deficient type II transforming growth factor beta (TGFbeta) receptor leads to paradoxical activation of TGFbeta signaling pathways with fibrosis in transgenic mice. J Biol Chem 278(27): 25109-25119, 2003. PMID: 12707256. DOI: 10.1074/jbc.M300636200

19 Ye Q, Chen B, Tong Z, Nakamura S, Sarria R, Costabel U and Guzman J: Thalidomide reduces IL-18, IL- 8 and TNF- $\alpha$ release from alveolar macrophages in interstitial lung disease. Eur Respir J 28(4): 824-831, 2006. PMID: 16837501. DOI: $10.1183 / 09031936.06 .00131505$ 
20 Huaux F, Liu T, McGarry B, Ullenbruch M and Phan SH: Dual roles of IL-4 in lung injury and fibrosis. J Immunol 170(4): 2083-2092, 2003. PMID: 12574379. DOI: 10.4049/jimmunol. 170.4 .2083

21 Borthwick LA, Wynn TA and Fisher AJ: Cytokine mediated tissue fibrosis. Biochim Biophys Acta 1832(7): 1049-1060, 2013. PMID: 23046809. DOI: 10.1016/j.bbadis.2012.09.014

22 Baroni GS, D'Ambrosio L, Curto P, Casini A, Mancini R, Jezequel AM and Benedetti A: Interferon gamma decreases hepatic stellate cell activation and extracellular matrix deposition in rat liver fibrosis. Hepatology 23(5): 1189-1199, 1996. PMID: 8621153. DOI: $10.1002 /$ hep.510230538

23 Kobayashi T, Tanaka K, Fujita T, Umezawa H, Amano H, Yoshioka K, Naito Y, Hatano M, Kimura S, Tatsumi K and Kasuya Y: Bidirectional role of IL-6 signal in pathogenesis of lung fibrosis. Respir Res 16: 99, 2015. PMID: 26289430. DOI: 10.1186/s12931-015-0261-Z

24 Lo Re S, Lison D and Huaux F: CD4+ T lymphocytes in lung fibrosis: diverse subsets, diverse functions. J Leukoc Biol 93(4): 499-510, 2013. PMID: 23159927. DOI: 10.1189/jlb.0512261
25 Xin X, Yao D, Zhang K, Han S, Liu D, Wang H, Liu X, Li G, Huang $\mathrm{J}$ and Wang $\mathrm{J}$ : Protective effects of Rosavin on bleomycin-induced pulmonary fibrosis via suppressing fibrotic and inflammatory signaling pathways in mice. Biomed Pharmacother 115: 108870, 2019. PMID: 31026730. DOI: 10.1016/j.biopha.2019.108870

26 Seger S, Stritt M, Vezzali E, Nayler O, Hess P, Groenen PMA and Stalder AK: A fully automated image analysis method to quantify lung fibrosis in the bleomycin-induced rat model. PLoS One 13(3): e0193057, 2018. PMID: 29547661. DOI: 10.1371/ journal.pone. 0193057
Received June 8, 2019

Revised July 1, 2019

Accepted July 4, 2019 